\title{
Underestimating hypoactive delirium?
}

\author{
Haruhiko Hoshino ${ }^{1,2}\left(\right.$ ] Jun Watanabe ${ }^{3,4,5} \cdot$ Masahiro Banno $^{5,6,7}$
}

Received: 4 March 2020 / Accepted: 3 May 2020 / Published online: 2 August 2020

(c) Japanese Society of Anesthesiologists 2020

To the Editor:

We have read with interest the article by Eun-Ah et al. [1], but feel that two aspects of this study must be clarified and discussed.

First, the authors may have underestimated hypoactive delirium, as this is often misdiagnosed and mistreated due to inconsistencies in the diagnosis itself. Delirium subtypes were designated as hypoactive, hyperactive, or mixed based on observed behavior and Emergence Delirium (ED) was rated using a four-point scale and the Pediatric Anesthesia Emergence Delirium tool, both of which cannot assess hypoactive delirium [2]. Consequently, raters could not properly assess hypoactive and mixed delirium. To correctly assess pediatric delirium, the Cornell Assessment Pediatric Delirium or the PreSchool Confusion Assessment Method for the ICU should have been used [3].

This comment refers to the article available online at https://doi. org/10.1007/s00540-019-02705-6.

Haruhiko Hoshino

haruhiko.hoshino@outlook.com

1 Department of Emergency and Critical Care Medicine, Faculty of Medicine, University of Tsukuba, Tsukuba, Ibaraki, Japan

2 Department of Nursing, Pediatric Intensive Care Unit, University of Tsukuba Hospital, Tsukuba, Ibaraki, Japan

3 Department of Surgery, Tottori Prefectural Central Hospital, 730 Ezu, Tottori, Tottori 680-0901, Japan

4 Center for Community Medicine, Jichi Medical University, 3311-1 Yakushiji, Shimotsuke, Tochigi 329-0498, Japan

5 Systematic Review Workshop Peer Support Group (SRWS-PSG), Osaka, Japan

6 Department of Psychiatry, Seichiryo Hospital, Tsurumai 4-16-27, Showa-ku, Nagoya 466-0064, Japan

7 Department of Psychiatry, Nagoya University Graduate School of Medicine, Tsurumai-cho 65, Showa-ku, Nagoya 466-8560, Japan
Finally, the results of this study do not support the conclusion as there was no placebo group. Hence, it is unclear that dexmedetomidine and midazolam are efficacious in preventing ED.

\section{References}

1. Eun-Ah C, Yun-Byeong C, Jae-Geum S, Jin-Hee A, Sung HL, Kyoung-Ho R. Comparison of single minimum dose administration of dexmedetomidine and midazolam for prevention of emergence delirium in children: a randomized controlled trial. $\mathrm{J}$ Anesth. 2020;34:59-655.

2. Traube C, Silver G, Kearney J, Patel A, Atkinson TM, Yoon MJ, Halpert S, Augenstein J, Sickles LE, Li C, Greenwald B. Cornell Assessment of Pediatric Delirium: a valid, rapid, observational tool for screening delirium in the PICU. Crit Care Med. 2014;42:656-63.

3. Dechnik A, Traube C. Delirium in hospitalised children. Lancet Child Adolesc Health. 2020;S2352-4642(19):30377-83.

Publisher's Note Springer Nature remains neutral with regard to jurisdictional claims in published maps and institutional affiliations. 